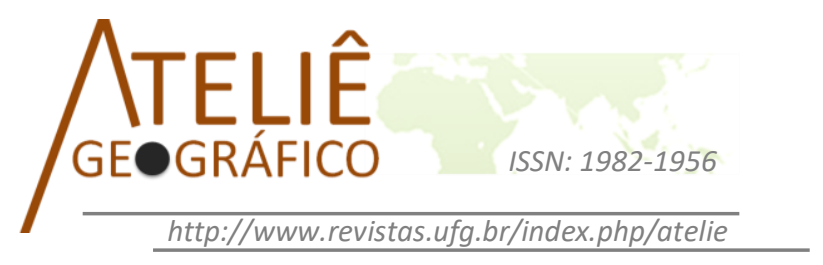

\title{
Vulnerabilidade socioambiental em bacias hidrográficas no Setor Leste Metropolitano de Fortaleza, Ceará
}

\author{
Environmental vulnerability in river basin in the Sector \\ Metropolitan Fortaleza East, Ceara
}

\section{Vulnerabilidad ambiental en la cuenca hidrográfica en el Sector del Este de la Región Metropolitana de Fortaleza, Ceará}

\author{
Emanuel Lindemberg Silva Albuquerque \\ Universidade Federal do Piauí \\ lindemberg@ufpi.edu.br \\ Cleyber Nascimento de Medeiros \\ Instituto de Pesquisa e Estratégia Econômica do Ceará \\ cleyber.medeiros@ipece.ce.gov.br
}

\begin{abstract}
Resumo
Ao considerar que as inovações geotecnológicas vêm potencializando novos estudos na contemporaneidade, constata-se que os preceitos da geoinformação possuem uma aplicação efetiva na abordagem de bacias hidrográficas. Dessa forma, o estudo em pauta objetiva mapear a vulnerabilidade socioambiental nas bacias hidrográficas costeiras dos rios Catú, Caburé, Caponga Funda, Caponga Roseira e Mal Cozinhado, localizadas no setor leste da Região Metropolitana de Fortaleza, estado do Ceará. A fundamentação teórico-metodológica encontra-se nos estudos sistêmicos, baseada no viés da concepção indissociável entre natureza e sociedade. O Índice de Vulnerabilidade Socioambiental foi estruturado a partir da análise integrada das condições ecodinâmicas (Vulnerabilidade Ambiental), em conjunto com a Vulnerabilidade Socioeconômica (Índice de Desenvolvimento Humano Municipal). Os resultados alcançados no estudo retratam que $82,27 \%$ da área é classificada na faixa de baixa ou média-baixa Vulnerabilidade Socioambiental e 17,73\% encontram-se na faixa de média-alta ou alta Vulnerabilidade. Conclui-se que na área em epígrafe há uma intensa pressão antropogênica sobre os sistemas ambientais, com destaque especial para as planícies fluviais, lacustres e litorânea.
\end{abstract}

Palavras-chave: Sistema Ambiental, Geotecnologias, Urbanização.

\section{Abstract}

When considering the geotecnology innovations come enhancing new studies in contemporary times, It notes that the geoinformation of precepts have an effective 
application in addressing watershed. Thus, the study aims to map the socio-environmental vulnerability in coastal watersheds of rivers Catú, Cabure, Caponga Funda, Caponga Roseira and Mal Cozinhado, located in the eastern sector of the metropolitan area of Fortaleza, state of Ceara. The theoretical-methodological foundation lies in the systemic studies, based on the bias of the indivisible conception of nature and society. The Environmental Vulnerability Index was structured from the integrated analysis of the ecodynamics conditions (Environmental Vulnerability), together with the Socio-Economic Vulnerability (Municipal Human Development Index). The results achieved in this study portray that $82.27 \%$ of the area is classified in the low zone or medium-low Environmental Vulnerability and $17.73 \%$ are in the medium-high or high vulnerability zone. We conclude that in the area referred is very intense the anthropogenic pressure on environmental systems, with special emphasis on the river, lake and coastal plains.

Keywords: Environmental System, Geotecnology Urbanization.

\begin{abstract}
Resumem
Al considerar que las innovaciones geotecnológicas intensifican los nuevos estudios en la época contemporánea, se constata que los preceptos de la geoinformación tienen una aplicación efectiva en el abordaje de las cuencas hidrográficas. Así, el estudio apunta a mapear la vulnerabilidad socio-ambiental en las cuencas costeras de los ríos Catú, Caburé, Caponga Funda, Caponga Roseira y Mal Cozinhado, ubicadas en el sector oriental de la zona metropolitana de Fortaleza, estado de Ceará. El fundamento teórico-metodológico se encuentra en los estudios sistémicos, basado en el sesgo de la concepción indivisible de la naturaleza y la sociedad. El Índice de Vulnerabilidad Ambiental se estructuró a partir del análisis integrada de las condiciones ecodinâmicas (Vulnerabilidad Ambiental), junto con la Vulnerabilidad Socioeconómica (Índice de Desarrollo Humano Municipal). Los resultados obtenidos en este estudio retractan que $82,27 \%$ de la superficie se clasifica en el zona bajo o medio-bajo Vulnerabilidad Ambiental y 17,73\% están en la zona de vulnerabilidad mediaalta o alta. Llegamos a la conclusión de que en el ámbito mencionado anteriormente hay una intensa presión antropogénica sobre los sistemas ambientales, con especial énfasis en el río, el lago y las llanuras costeras.
\end{abstract}

Palabras clave: Sistema Ambiental, Geotecnológicas, Urbanización.

\title{
Introdução
}

Desde a última década do século XX, o campo de ação da pesquisa geográfica vem sendo influenciado por novos paradigmas do mundo moderno, sobretudo, quando atrelado ao uso das tecnologias da geoinformação. Nesse sentido, as inovações geotecnológicas vêm desvendando, de forma efetiva, as reais potencialidades de avaliar a vulnerabilidade socioambiental na contemporaneidade.

Conforme Cunha (2001), para que as ações do poder público sejam orientadas às necessidades da população e compatíveis com a realidade local, é importante conhecê-las segundo as suas potencialidades e limitações ambientais, sociais e econômicas. Nesse sentido, a análise da vulnerabilidade socioambiental surge como um importante delineador das condições organizacionais do espaço geográfico, por abordar o estudo integrado da paisagem sob a ótica sistêmica.

Destaca-se que a integralização e espacialização dos dados e informações georreferenciadas tornaram-se um procedimento fundamental no estudo do espaço geográfico, constatando-se que a análise geoespacial apresenta-se como um 
procedimento adequado na perspectiva de orientar o ordenamento territorial (ALBUQUERQUE, et al., 2015).

No intuito de agregar as informações das variáveis que compõem a vulnerabilidade socioambiental ao mesmo nível geográfico, adota-se nesta pesquisa o recorte espacial por meio das Unidades Hidrográficas de Planejamento (UHPs), seguindo a proposta originalmente apresentada por Zanella et al. (2013).

Salienta-se que a UHP, conforme os autores citados, representa um recorte territorial que adequa o limite topográfico da bacia hidrográfica, que é o recorte espacial adotado nesta pesquisa, aos setores censitários do setor leste metropolitano de Fortaleza, estado do Ceará. Neste sentido, visa avaliar não somente os elementos ambientais relacionados ao limite da bacia hidrográfica, mas sim o conjunto integrante das variáveis que compõem a situação socioeconômica.

Corrobora-se que esta perspectiva de análise territorial segue os princípios delineados por Laura (2004), reconhecendo que a bacia hidrográfica é concebida como um sistema complexo, aberto, cujos elementos biológicos, sociais e econômicos se encontram em estreita inter-relação, com fluxos, influências e linhas de ação que atravessam suas fronteiras, como é o caso dos setores censitários adotados no Censo Demográfico (IBGE, 2010).

Neste sentido, esta pesquisa tem como foco principal avaliar a vulnerabilidade socioambiental das bacias hidrográficas costeiras dos rios Catú, Caburé, Caponga Funda, Caponga Roseira e Mal Cozinhado, por meio do Índice de Vulnerabilidade Socioambiental (IVSA) agregados as UHPs, na perspectiva de contribuir como subsídio ao ordenamento territorial do setor leste da Região Metropolitana de Fortaleza (RMF).

\section{Localização e Caracterização da área de estudo}

A área em estudo compreende as bacias hidrográficas costeiras dos rios Catú $\left(161,12 \mathrm{~km}^{2}\right)$, Caburé $\left(90,41 \mathrm{~km}^{2}\right)$, Caponga Funda $\left(61,39 \mathrm{~km}^{2}\right)$, Caponga Roseira $\left(73,49 \mathrm{~km}^{2}\right)$ e Mal Cozinhado $\left(408,47 \mathrm{~km}^{2}\right)$, envolvendo em todo ou em parte os municípios de Aquiraz, Cascavel, Horizonte, Pacajus e Pindoretama, totalizando um recorte territorial que compreende 794,88 km² (Figura 1).

Devido à proximidade com Fortaleza, capital do estado do Ceará, o setor leste da Região Metropolitana de Fortaleza (RMF) vem apresentando forte incremento populacional e econômico (industrial, turístico e imobiliário), sobretudo, nos últimos 30 anos, possuindo assim um significativo volume de variáveis/indicadores que na maioria das vezes não são abordados, mormente, quando se analisa os recursos naturais das bacias hidrográficas costeiras por meio da vulnerabilidade socioambiental. 


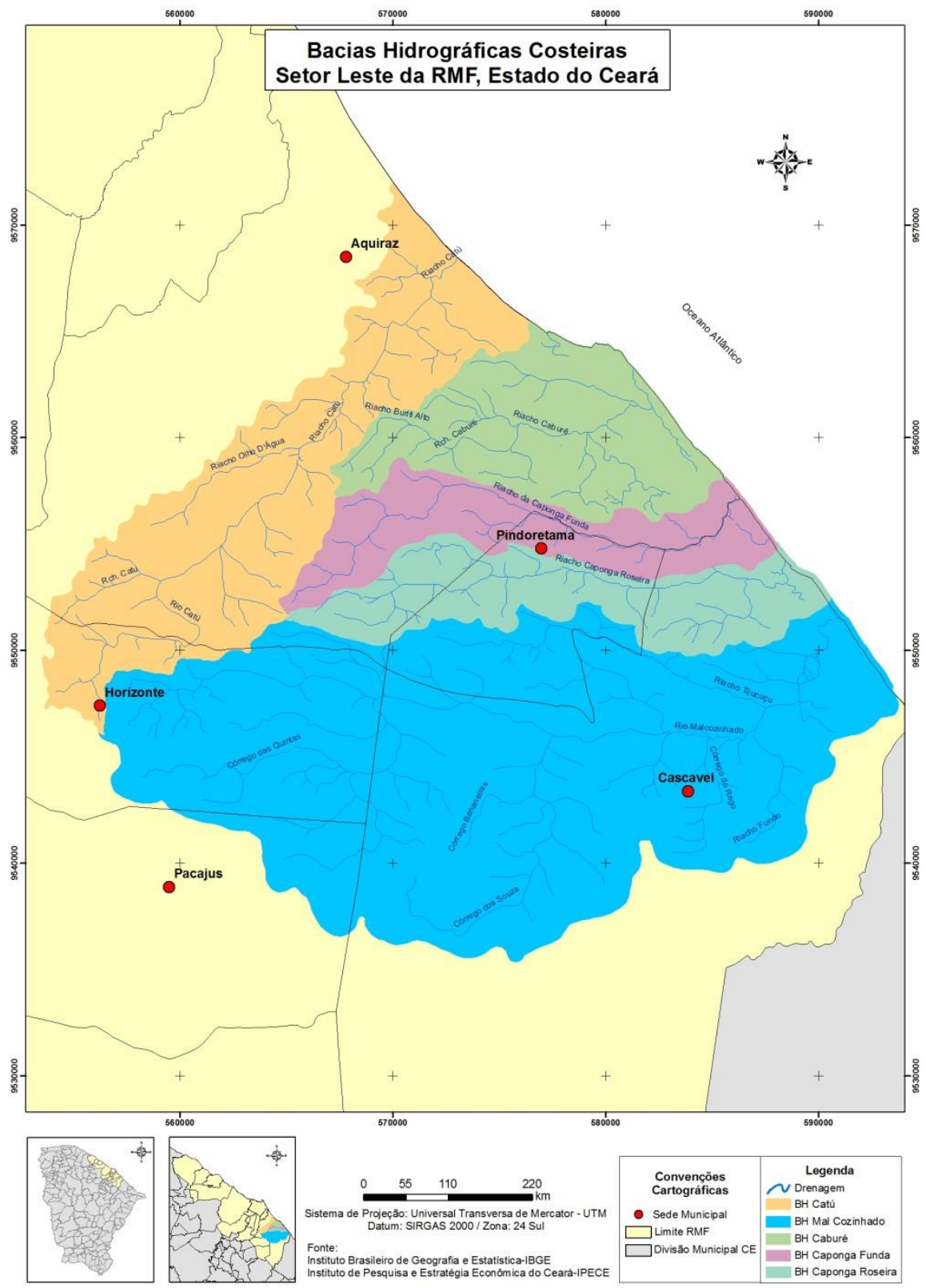

Figura 1 - Mapa de localização da área de estudo

Do ponto de visa populacional, constata-se que os municípios mencionados têm apresentado nos últimos anos um acentuado crescimento. Tendo como referência o total populacional da área, o mesmo correspondia a 155.337 habitantes em 1991, 
210.409 no ano 2000 e 274.478 habitantes em 2010, alcançando uma elevação relativa de $23,40 \%$ no derradeiro decênio, bem superior à média do Ceará, que foi de $13,75 \%$, como pode ser avaliada na Tabela 1.

Tabela 1 - População residente nos municípios inseridos nas bacias hidrográficas em apreço $1991 / 2000 / 2010$

\begin{tabular}{l|c|c|c|c|c}
\hline \multirow{2}{*}{ Municípios } & \multicolumn{3}{|c|}{ População Residente } & \multicolumn{2}{c}{ Taxa geométrica de crescimento anual } \\
& $\mathbf{1 9 9 1}$ & $\mathbf{2 0 0 0}$ & $\mathbf{2 0 1 0}$ & $\mathbf{1 9 9 1 / 2 0 0 0}$ & $\mathbf{2 0 0 0 / 2 0 1 0}$ \\
\cline { 2 - 6 } & 46.305 & 60.469 & 72.628 & 3,01 & 1,85 \\
\hline Aquiraz & 46.507 & 57.129 & 66.142 & 2,31 & 1,48 \\
\hline Cascavel & 18.283 & 33.790 & 55.187 & 7,06 & 5,03 \\
\hline Horizonte & 31.800 & 44.070 & 61.838 & 3,69 & 3,45 \\
\hline Pacajus & 12.442 & 14.951 & 18.683 & 2,06 & 2,25 \\
\hline Pindoretama & $\mathbf{2 . 4 7 3 . 2 9 7}$ & $\mathbf{3 . 0 5 6 . 7 6 9}$ & $\mathbf{3 . 6 1 5 . 7 6 7}$ & $\mathbf{2 , 3 8}$ & $\mathbf{1 , 6 9}$ \\
\hline RMF (total) & $\mathbf{6 . 3 6 6 . 6 4 7}$ & $\mathbf{7 . 4 3 0 . 6 6 1}$ & $\mathbf{8 . 4 5 2 . 3 8 1}$ & $\mathbf{1 , 7 3}$ & $\mathbf{1 , 3 0}$ \\
\hline Ceará (total) & $\mathbf{6}$ &
\end{tabular}

Fonte: IBGE (Censos Demográficos). Elaboração dos autores.

Dessa forma, considerando a série histórica, nota-se que os dados populacionais estão relacionados, notadamente, ao crescimento expressivo e vertiginoso da população que veio, principalmente, do interior cearense (PEREIRA JÚNIOR, 2005), em busca de melhores condições de vida na RMF.

Para Silva (2009), a RMF vem experimentando transformações rápidas em sua estrutura e fisionomia urbana, com mudanças substanciais nos novos municípios que são agregados a essa Macrorregião de Planejamento, com destaque especial para o significativo processo de urbanização/crescimento populacional que vem se intensificando a cada ano.

Neste contexto, corrobora-se que a dinâmica espacial deve ser entendida como um conjunto de eventos interconectados e estabelecidos, em que as suas interações refletem a estrutura da realidade, ou seja, é um processo espacial com dimensão temporal, como bem destaca Anjos (2008).

\section{Vulnerabilidade Socioambiental: aspectos teóricos da abordagem}

O conceito de vulnerabilidade, em sua visão generalizada, corresponde a um conjunto de processos e condições resultantes de fatores físicos, biológicos, sociais, econômicos e políticos que aumentam a suscetibilidade ao impacto de um perigo com vistas à redução do bem-estar (TOMINAGA, 2009).

Ao considerar esse preceito, diversos autores desenvolveram parâmetros e índices relacionados à representação cartográfica da vulnerabilidade, sobretudo, quando se analisa o viés ambiental, social e socioambiental de uma determinada porção geográfica, tanto de forma isolada quanto integrada. 
Zanella et al. (2013) corrobora que o termo vulnerabilidade vem sendo utilizado para expressar a instabilidade dos ambientes, sendo que tal assertiva encontrase em consonância com o viés delineado por Tricart (1977), em virtude que a vulnerabilidade consiste no maior ou menor estágio de estabilidade e instabilidade dos elementos físicos e biológicos frente ao processo de uso e ocupação do espaço geográfico.

No entanto, todos esses preceitos, em síntese, encontram-se atrelados aos princípios do ordenamento territorial. Para Grigio (2003), faz-se necessário conhecer a forma como o ambiente reage às pressões antrópicas impostas, assim como o grau de suporte a essas influências frente aos mais diversos cenários degradacionais e/ou conservacionistas do espaço geográfico.

Dessa forma, ao invés de abordar a vulnerabilidade ambiental e social de forma segmentada, para um posterior cruzamento das informações obtidas, optou-se, metodologicamente para este estudo, trabalhar com o conceito de vulnerabilidade socioambiental, tendo em vista que o mesmo agrega as variáveis ambientais e socioeconômicas num todo integrado.

Deschamps (2004) corrobora que a vulnerabilidade socioambiental corresponde à ocorrência simultânea da vulnerabilidade social e ambiental em um mesmo território ou para uma mesma população. Esse conceito se aproxima dos preceitos de Alves (2005), em que o autor cita que a vulnerabilidade socioambiental caracteriza-se como sendo uma sobreposição de fatores sociais e ambientais em um determinado território, num dado recorte temporal.

Para Medeiros (2014), a vulnerabilidade socioambiental corresponde a um local onde coexistem ambientes e populações em situação de risco, expondo os indivíduos às adversidades decorrentes de fenômenos naturais e sociais, tais como inundações ou enchentes, deslizamentos de terra, soterramentos, erosão marinha, secas recorrentes, acometimentos de doenças, perdas de emprego, entre outras variáveis.

\section{Procedimentos Metodológicos e Operacionais}

Inicialmente foi realizado um estudo descritivo quali-quantitativo no sentido de identificar e descrever os aspectos geoambientais dos sistemas naturais que compõem as bacias hidrográficas costeiras em epígrafe. Nesse mesmo viés, trabalhouse com os dados ambientais, sociais e econômicos ao nível de setores censitários, por meio da análise integrada.

Para trabalhar com essa delimitação ao nível dos setores censitários, optou-se por definir a área de estudo em Unidades Hidrográficas de Planejamento (UHPs). Dessa forma, foram estruturadas 5 (cinco) UHPs, a saber: UHP Catú; UHP Caburé; UHP Caponga Roseira; UHP Caponga Funda e; UHP Mal Cozinhado.

É importante considerar que o padrão espacial dos elementos socioambientais das UHPs foram delimitados em função do limite geográfico das bacias hidrográficas 
costeiras, utilizando-se critérios para adequá-los aos polígonos dos setores censitários, tendo em vista que o limite da bacia hidrográfica é diferente do limite políticoadministrativo.

Neste contexto, foram considerados integrantes das UHPs todos os setores censitários que apresentam pelo menos 50\% de sua área no interior do polígono da bacia (Figura 2). Este mesmo procedimento operacional segue a metodologia adotada por Guimarães (2008), Zanella et al. (2013) e Albuquerque (2015).

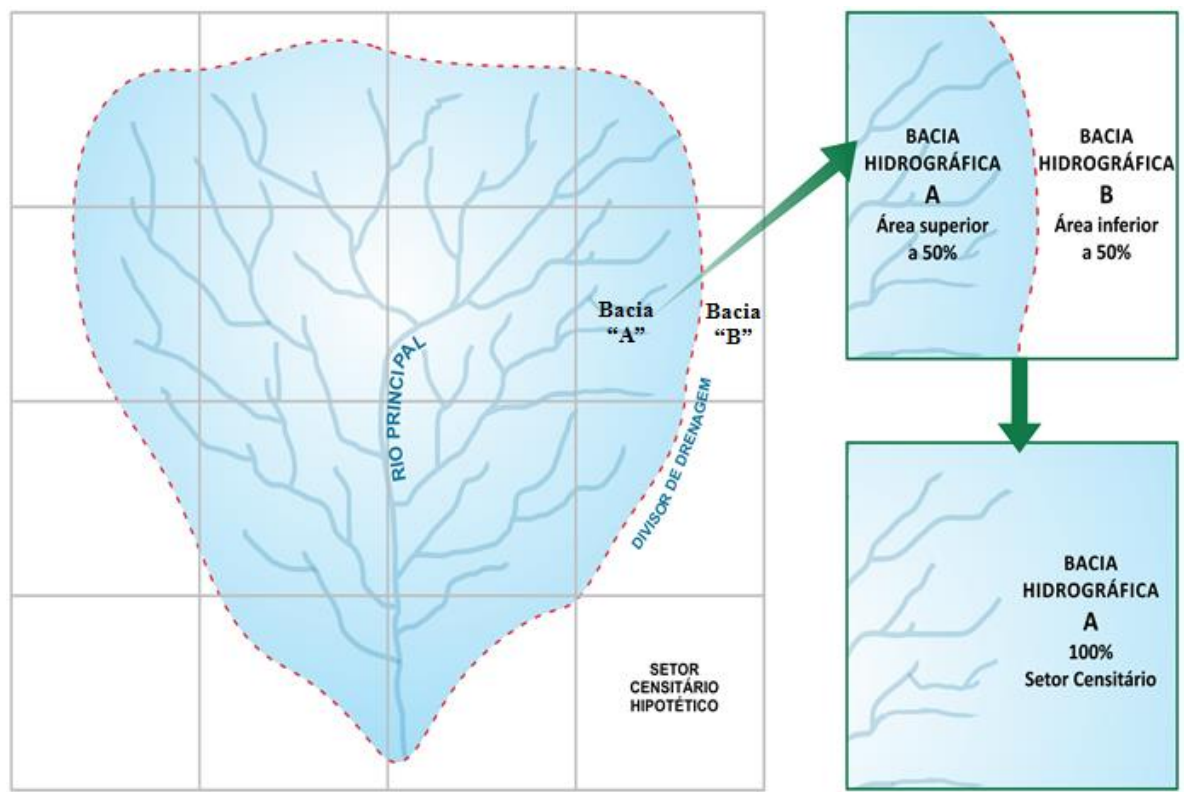

Figura 2 - Representação esquemática do procedimento metodológico de padronização dos setores censitários ao limite das bacias hidrográficas.

Fonte: Elaboração dos autores.

Por sua vez, os limites dos setores censitários foram adaptados nas áreas de borda da bacia hidrográfica, especificamente, nos divisores de água das bacias dos rios Catú e Mal Cozinhado. Ressalta-se que por meio da interpretação de imagens de satélite e pelos trabalhos de campo, bem como pela análise dos dados populacionais destes setores censitários adaptados, identificou-se que os mesmos possuem baixa densidade populacional, não impactando, portanto, nos resultados almejados.

Aliado a esses fatores, justifica-se que o recorte espacial dos mencionados setores aos limites das bacias hidrográficas possibilita a análise integrada dos indicadores ambientais e socioeconômicos ao nível da UHP e, consequentemente, possibilita a concepção do Índice de Vulnerabilidade Socioambiental (IVSA), já que o 
mesmo aborda na mesma célula espacial as características ambientais, sociais e econômicas.

Com base neste procedimento, estruturaram-se as UHPs conforme a Figura 3. A importância de se trabalhar com esse tipo de unidade é pela perspectiva de ter um maior, e mais preciso, arcabouço de dados ambientais e socioeconômicos, tendo em vista a sua precisão cartográfica quando se compara com a realidade geoambiental, o que possibilita avaliar de forma sistêmica as condições socioambientais presentes no espaço geográfico.

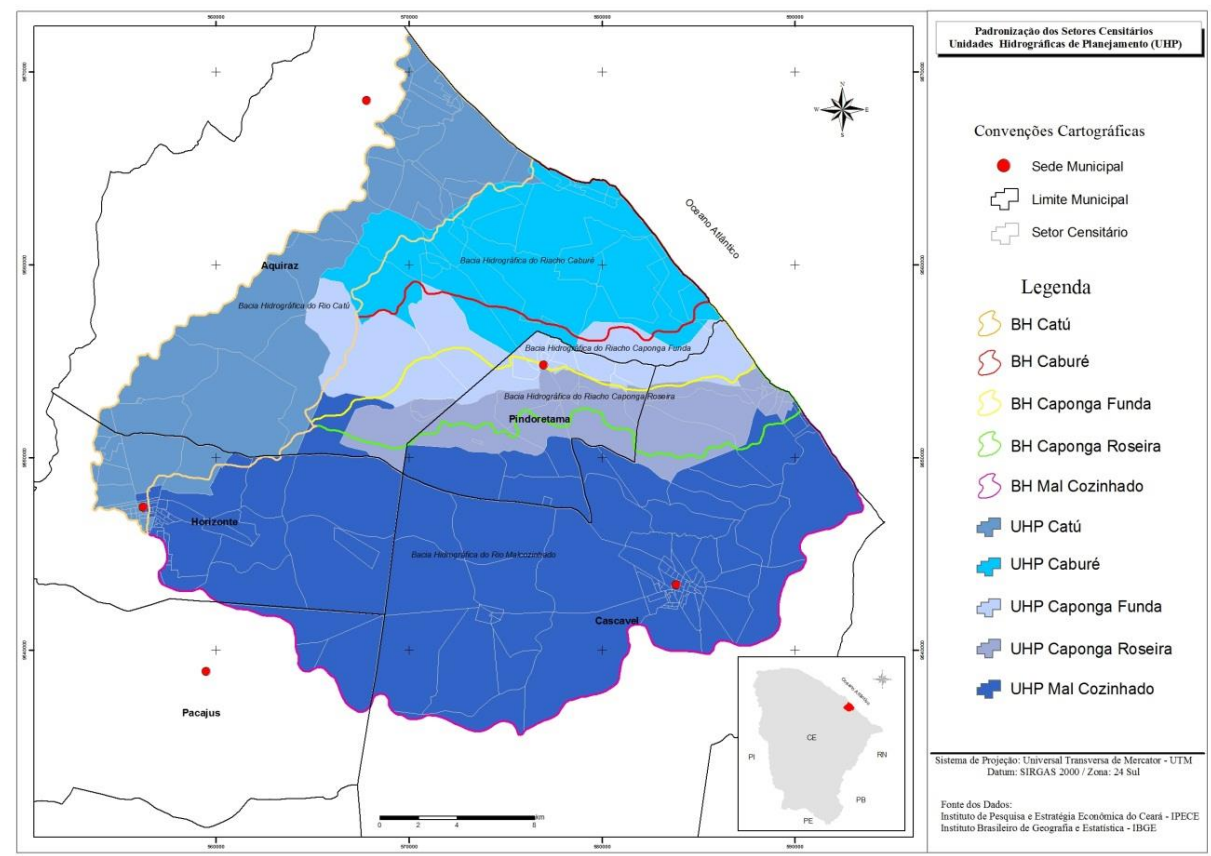

Figura 3 - Padronização dos setores censitários às UHPs

Destaca-se que o Índice de Vulnerabilidade Socioambiental (IVSA) é composto, em essência, por variáveis ambientais e socioeconômicas. Cita-se que a dimensão ambiental se refere às informações sobre as condições ecodinâmicas dos sistemas ambientais presentes nas bacias hidrográficas costeiras em epígrafe, obtidas por meio da compartimentação geoambiental realizada por Albuquerque (2015). Este trabalho considerou as características do ambiente natural por meio dos preceitos das potencialidades e fragilidades dos sistemas ambientais (SOUZA, 2000) e pelas condições ecodinâmicas (TRICART, 1977).

A dimensão socioeconômica foi caracterizada por meio do Índice de Desenvolvimento Humano Municipal (IDHM). Frisa-se que o IDHM foi elaborado pelo Programa das Nações Unidas (PNUD, 2014), em parceria com a Fundação João 
Pinheiro (FJP), o Instituto de Pesquisa Econômica Aplicada (IPEA) e o Instituto de Pesquisa e Estratégia Econômica do Ceará (IPECE), abrangendo os municípios da Região Metropolitana de Fortaleza (RMF) e tendo como recorte geográfico as Unidades de Desenvolvimento Humano (UDH), as quais correspondem aos setores censitários utilizados no Censo Demográfico (IBGE, 2010).

Conforme o PNUD (2013), o desenvolvimento humano corresponde ao processo de ampliação das liberdades dos indivíduos com relação às suas capacidades e às oportunidades a seu dispor, de forma que cada pessoa possa escolher a vida que deseja ter, visando à melhora ou a manutenção das condições de seu bem-estar.

Ao considerar as dimensões expostas, apresenta-se a seguir a descrição dos indicadores que compõem o IVSA (Quadro 1).

\begin{tabular}{|c|c|c|}
\hline DIMENSÖES & INDICADORES & DESCRIÇÃO \\
\hline \multirow{3}{*}{ AMBIENTAL } & Ambientes Estáveis & $\begin{array}{l}\text { São ambientes com estabilidade morfogenética antiga; solos espessos e } \\
\text { bem evoluídos; predomínio da pedogênese sobre os processos } \\
\text { morfogenéticos; cobertura vegetal tendendo a condições climáxicas, } \\
\text { prevalecendo a fitoestabilidade, com vulnerabilidade ambiental muito baixa. }\end{array}$ \\
\hline & $\begin{array}{l}\text { Ambientes de } \\
\text { (Intergrades) }\end{array}$ & $\begin{array}{l}\text { Corresponde a ação simultânea dos processos morfogenéticos e } \\
\text { pedogenéticos; incidência moderada dos processos erosivos areolares; } \\
\text { predominância da pedogênese evidencia tendência à estabilidade; } \\
\text { predominância da morfogênese indica tendência à instabilidade, com } \\
\text { vulnerabilidade ambiental de moderada a alta. }\end{array}$ \\
\hline & Ambientes Fortemente Instáveis & $\begin{array}{l}\text { Há intensificação dos processos morfogenéticos; relevos fortemente } \\
\text { dissecados e vertentes com declives ingrimes; condições climáticas } \\
\text { agressivas; baixa capacidade protetora exercida pela vegetação aos solos; } \\
\text { solos erodidos com pedogênese praticamente nula; nítida preponderância } \\
\text { da morfogênese sobre a pedogênese, com vulnerabilidade ambiental alta. }\end{array}$ \\
\hline $\begin{array}{l}\text { SOCIAL E } \\
\text { ECONÔMICO }\end{array}$ & $\begin{array}{l}\text { İndice de Desenvolvimento } \\
\text { Humano Municipal (IDHM) }\end{array}$ & $\begin{array}{l}\text { O İndice de Desenvolvimento Humano Municipal (PNUD, 2013) } \\
\text { corresponde a média geométrica dos indices das dimensões Renda, } \\
\text { Educação e Longevidade, com pesos iguais, variando no intervalo de } 0 \text { a } 1 \text {. } \\
\text { O İndice sintético da dimensão Educação é obtido através da média } \\
\text { geométrica do subindice de frequência de crianças e jovens à escola, com } \\
\text { peso de } 2 / 3 \text {, e do subindice de escolaridade da população adulta, com peso } \\
\text { de } 1 / 3 \text {. O İndice da dimensão Longevidade é obtido a partir do indicador } \\
\text { Esperança de vida ao nascer. O Índice da dimensão Renda é obtido a partir } \\
\text { do indicador Renda per capita. }\end{array}$ \\
\hline
\end{tabular}

Fonte: Elaboração dos autores.

Na perspectiva de cruzar as variáveis ambientais e socioeconômicas, recorreuse a análise integrada para a combinação dos mapas de vulnerabilidade ambiental (derivado da compartimentação ambiental) e socioeconômica (medida por meio do IDHM), almejando gerar o Índice de Vulnerabilidade Socioambiental (IVSA), dentro de uma perspectiva sistêmica e holística. A operacionalização foi realizada com base em Sistemas de Informações Geográficas (SIGs), especificamente o programa ArcGIS $^{\circledR}$, versão Trial-FREE.

Vale salientar que, inicialmente, foram atribuídos pesos compensatórios a cada classe temática da vulnerabilidade ambiental, especificamente: 0,33 para a classe de vulnerabilidade baixa; 0,66 para a classe de vulnerabilidade média e 1,00 para a classe de vulnerabilidade alta, considerando o intervalo entre 0 e 1. 
Ressalta-se que estes pesos foram adotados com base na ecodinâmica (TRICART, 1977; SOUZA, 2000) dos sistemas ambientais. Salienta-se que quanto mais próximo de 1 estiver o índice, mais vulnerável é o local em termos ambientais.

Na sequência, utilizando a extensão Geoprocessing Wizard (função Union) do software mencionado, foi realizado o cruzamento das entidades gráficas e não gráficas (atributos) dos produtos gerados (arquivos no formato shapefile). Como se inseriu os pesos de vulnerabilidade de cada classe dos mapas na tabela de atributos, ao final da união dos mapas dispunham-se dos graus de vulnerabilidade ambiental e socioeconômica (IDHM) em apenas um mapa, variando no intervalo de 0 a 1 . Salientase que o Índice de Vulnerabilidade Socioambiental (IVSA) é inversamente proporcional ao Índice de Desenvolvimento Humano Municipal (IDHM).

Neste contexto, menciona-se que a análise espacial empreendida através da função Union é justificada pela possibilidade da realização de ajustes importantes no momento do cruzamento dos mapas, pois são gerados novos polígonos (Figura 4) que agregam as informações gráficas e os atributos dos dois mapas em um só.

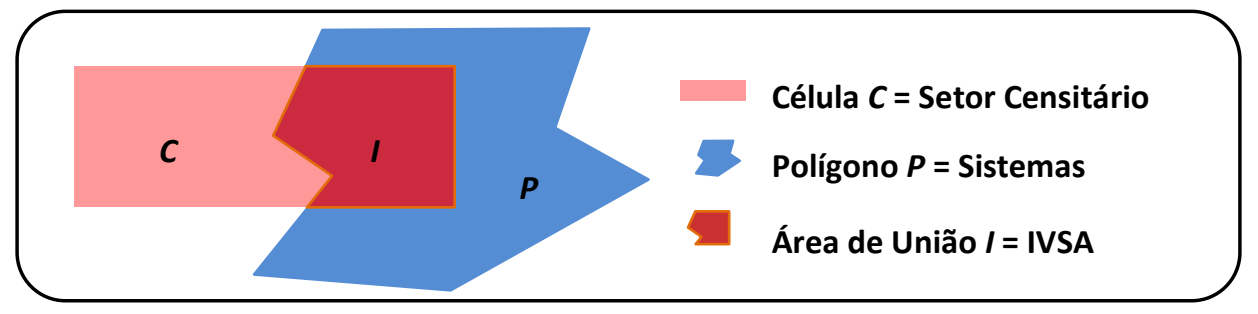

Figura 4 - Cruzamento de dois mapas usando a função de análise espacial Union. Fonte: Elaboração dos autores.

Vale destacar que o Índice de Vulnerabilidade Socioambiental (IVSA) é obtido a partir da média aritmética dos indicadores das dimensões: ambiental e socioeconômica. Menciona-se que após a definição do Índice, é realizada uma classificação das feições especificando quatro classes de vulnerabilidade, baseadas na média e na variabilidade (desvio-padrão) do IVSA.

Para tanto, foram estruturadas as seguintes classes: Baixa Vulnerabilidade (intervalo entre 0,000 - 0,3611), Média-Baixa Vulnerabilidade (intervalo entre 0,3612 - 0,5265), Média-Alta Vulnerabilidade (intervalo entre 0,5266 - 0,6919) e Alta Vulnerabilidade (intervalo entre 0,6920 - 1,0000), que são descritas a seguir:

i) Classe 1: Alta Vulnerabilidade, para valores superiores ao índice médio somado ao valor do desvio padrão;

ii) Classe 2: Média-Alta Vulnerabilidade, para valores maiores que o valor médio e menores que a média mais o valor do desvio padrão; 
iii) Classe 3: Média-Baixa Vulnerabilidade, para valores inferiores à média e superiores à média menos um desvio padrão;

iv) Classe 4: Baixa Vulnerabilidade, para índices com valores inferiores à média menos um desvio padrão.

Neste contexto, a vantagem da referida abordagem é dado pelo caráter prático em buscar compreender as condições socioambientais das bacias hidrográficas costeiras de forma integrada (Vulnerabilidade Ambiental + Vulnerabilidade Socioeconômica $=$ Vulnerabilidade Socioambiental), mesmo diante das diversidades e adversidades que se materializam no território.

\section{Resultados e discussões}

Por compilar multivariáveis, o Índice de Vulnerabilidade Socioambiental (IVSA), foi estruturado a partir da análise integrada das condições ecodinâmicas (Vulnerabilidade Ambiental), em conjunto com a Vulnerabilidade Socioeconômica (calculado por meio do Índice de Desenvolvimento Humano Municipal). Desta forma, tem-se que o IVSA varia no intervalo entre 0 e 1 , ou seja, quanto mais próximo de 1 , mais vulnerável tende a ser a subunidade hidrográfica de planejamento, que é materializada territorialmente por meio do setor censitário.

Ao considerar as características da compartimentação geoambiental, que derivou os atributos da Vulnerabilidade Ambiental, verifica-se que 81\% (ou 646,52 $\mathrm{km}^{2}$ ) da área é representada pela faixa de Baixa Vulnerabilidade (Ambientes Estáveis), sobretudo pela predominância do sistema ambiental dos tabuleiros pré-litorâneos, que possuem equilíbrio ecodinâmico. Em contrapartida, constata-se que 12,98\% (ou 103,17 $\mathrm{km}^{2}$ ) representam setores de Alta Vulnerabilidade Ambiental (Ambientes instáveis e fortemente instáveis), com destaque para os sistemas ambientais das planícies fluviais e lacustres (com 56,66 $\mathrm{km}^{2}$ ), das dunas móveis e dunas fixas (com 32,01 km²), da faixa de praia e terraços marinhos (com 11,62 $\mathrm{km}^{2}$ ), dos morros e cristas residuais (com 1,61 $\mathrm{km}^{2}$ ) e das planícies flúvio-marinhas (com 1,27 $\mathrm{km}^{2}$ ). Do ponto de vista da Vulnerabilidade Média (Ambientes de transição), merece destaque a área de transição tabuleiro pré-litorâneo/depressão sertaneja, que possui $17,48 \mathrm{~km}^{2}$ ou $2,20 \%$ da área em estudo. O restante da área compreende corpos d'águas $(3,82 \%)$, como pode ser visualizado na Figura 5.

De acordo com Souza (2000), a análise integrada do meio ambiente presume a consideração dos mecanismos que integram harmonicamente a natureza e a sociedade, a partir do viés de sua complexidade e heterogeneidade presente no território, concretizando assim a percepção do conjunto que está imbricada a análise socioambiental, sobretudo, nas áreas mais vulneráveis do ponto de vista de suas potencialidades e limitações. 


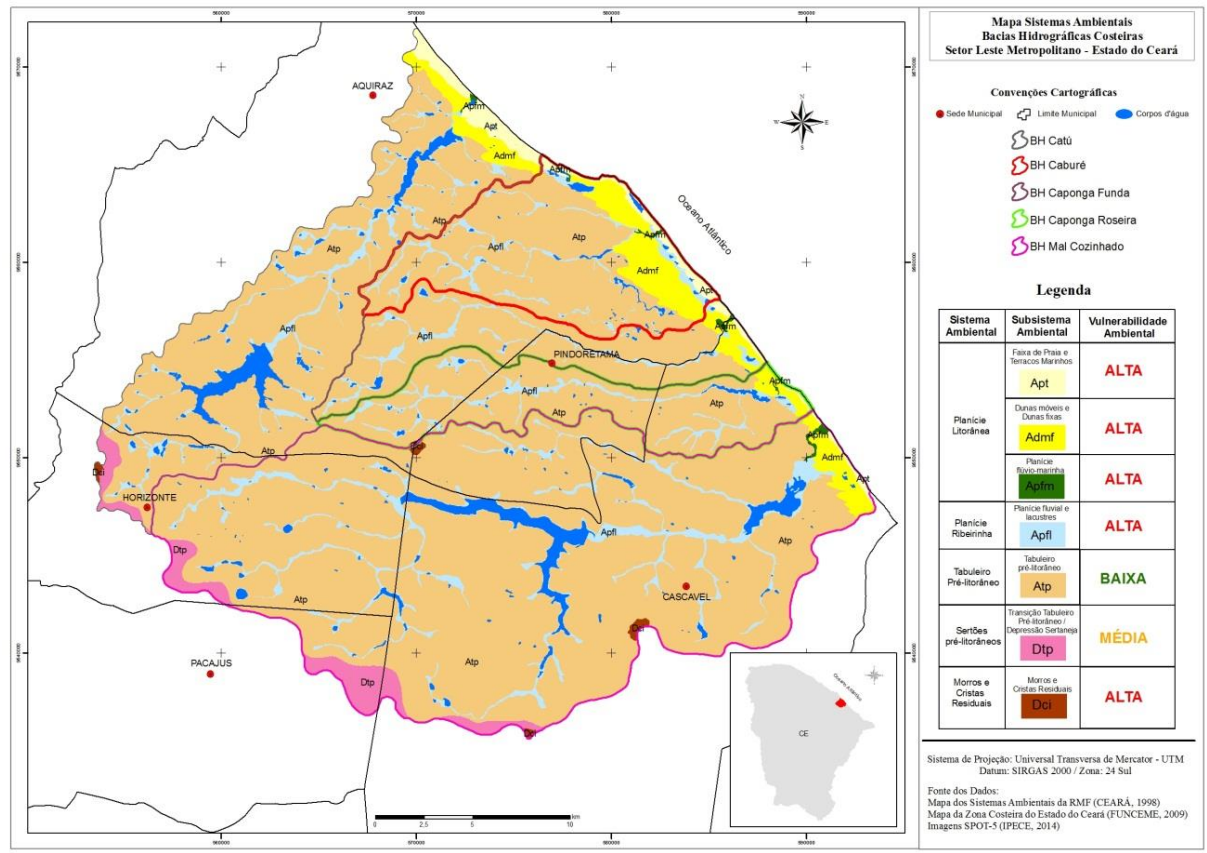

Figura 5 - Mapa de sistemas ambientais (Vulnerabilidade Ambiental) do setor leste metropolitano de Fortaleza, estado do Ceará

De posse das características ambientais, realizou-se a caracterização socioeconômica ao nível das Unidades Hidrográficas de Planejamento (UHPs) no setor leste da Região Metropolitana de Fortaleza (RMF), tendo como base, notadamente, os dados do Índice de Desenvolvimento Humano Municipal (IDHM), que avalia as condições socioeconômicas da população referente à renda, educação e longevidade (PNUD, 2013). A Figura 6 apresenta o IDHM, referente ao ano de 2010 (PNUD, 2014), no recorte espacial adotado no presente estudo.

Com base nas análises procedidas ao nível do IDHM, corrobora-se que as Unidades Hidrográficas de Planejamento (UHPs) representam um polo concentrador de riqueza e, consequentemente, de pobreza, que congrega uma multiplicidade de atores que atuam conforme os seus interesses e/ou necessidades. Em síntese, percebe-se que o desenvolvimento socioeconômico é concretizado de forma bastante desigual ao nível das UHPs, o qual reflete nos resultados quando se avalia a bacia hidrográfica como um todo integrado, tanto no urbano quanto no rural. 


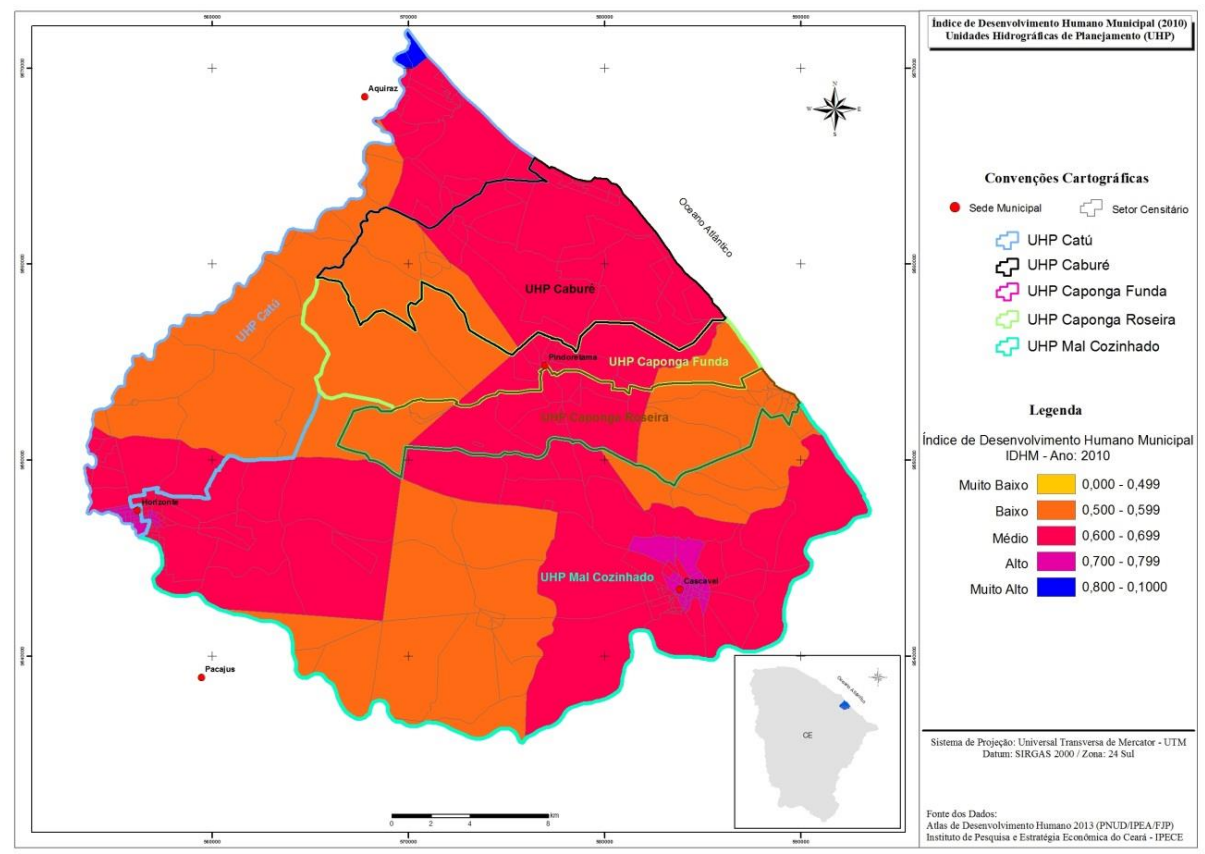

Figura 6 - IDHM (2010) do setor leste metropolitano de Fortaleza, Ceará

Ao considerar que o Índice de Vulnerabilidade Socioambiental (IVSA) foi estruturado e desenvolvido com vistas a compilar multivariáveis ao mesmo nível espacial, por meios das variáveis naturais (Vulnerabilidade Ambiental) e socioeconômicas (Vulnerabilidade Socioeconômica), tem-se que esse viés conceitual materializa as pressões que ocorrem no ambiente e, consequentemente, configuram-se nas causas primordiais dos problemas socioambientais (Vulnerabilidade Socioambiental).

De posse dos dados e informações obtidos por meio Índice de Vulnerabilidade Socioambiental (IVSA) ao nível das Unidades Hidrográficas de Planejamento (UHPs) dos rios Catú, Caburé, Caponga Funda, Caponga Roseira e Mal Cozinhado, estruturouse o Sistema de Informação Geográfica (SIG) na perspectiva de avaliar, de forma integrada, o IVSA no setor leste da Região Metropolitana de Fortaleza (RMF).

A seguir, apresenta-se na Tabela 2 a classificação do IVSA para o setor leste da RMF, exibindo as faixas do índice em consonância com sua área territorial $\left(\mathrm{em} \mathrm{km}^{2}\right)$ e o seu percentual (\%). Destaca-se que os setores mapeados como corpos d'água não entra no somatório do referido índice, já que o mesmo representa somente o espelho d'água ao nível das subunidades hidrográficas de planejamento. 
Tabela 2 - Índice de Vulnerabilidade Socioambiental (IVSA) nas UHPs

\begin{tabular}{l|l|l}
\hline \multicolumn{2}{c}{ IVSA - UHP do setor leste da RMF } \\
\hline Classificação & ÁREA $\left(\mathbf{k m}^{2}\right)$ & \% do Total \\
\hline Baixa Vulnerabilidade & 349,87 & 45,60 \\
\hline Média-Baixa Vulnerabilidade & 304,35 & 39,67 \\
\hline Média-Alta Vulnerabilidade & 73,67 & 9,60 \\
\hline Alta Vulnerabilidade & 39,30 & 5,12 \\
\hline TOTAL & $\mathbf{7 6 7 , 1 9}$ & $\mathbf{1 0 0}$ \\
\hline
\end{tabular}

Fonte: Elaboração dos autores.

Constata-se, em síntese, que aproximadamente $85 \%$, ou $654 \mathrm{~km}^{2}$ da área em estudo, compreendem setores de Baixa e Média-Baixa Vulnerabilidade, enquanto $15 \%$ são categorizados como de Média-Alta e Alta Vulnerabilidade. Destaca-se que o número elevado de setores com Baixa e Média-Baixa Vulnerabilidade é justificado, sobretudo, pela predominância do compartimento geoambiental dos tabuleiros prélitorâneos, em conformidade com uma população residente em setores censitários que são categorizados na faixa de melhores condições frente ao Índice de Desenvolvimento Humano Municipal (IDHM).

Com base nas análises precedidas, verifica-se que as áreas urbanas dos municípios de Cascavel, Horizonte (Figura 7) e Pindoretama, acrescida do setor leste do perímetro urbano de Aquiraz, encontram-se instalados, predominantemente, sobre os tabuleiros pré-litorâneo e apresentam as mais baixas vulnerabilidades socioambientais de acordo com o IVSA.

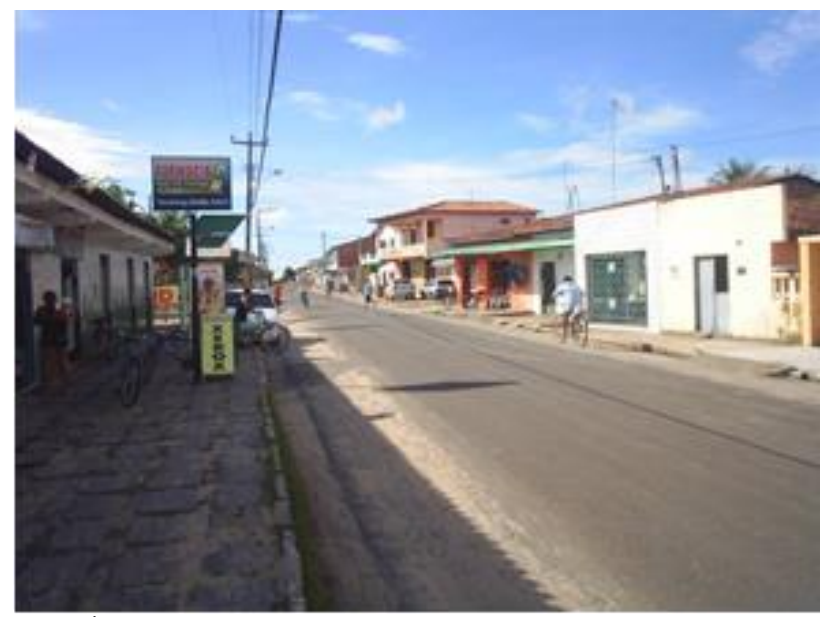

Figura 7: Área urbana inserida no tabuleiro pré-litorâneo, Horizonte (CE). Fonte: Autores (2014). Coordenada UTM / Datum SIRGAS 2000 / E= 556.149; N= 9.547.201 
Via de regra, corrobora-se que as áreas dos tabuleiros pré-litorâneos constituem os setores mais adequados para o adensamento urbano e a concentração demográfica, dada a topografia praticamente plana. Para tal uso e ocupação, devem ser levados em consideração no ordenamento territorial os aspectos vinculados ao saneamento básico e à implementação de infraestrutura urbana adequada, que ainda é deficitária em alguns setores.

Por outro lado, torna-se pertinente a conjunção de políticas públicas, por meio de ações efetivas, nas áreas de Média-Alta e Alta Vulnerabilidade, tendo em vista que esses setores são os mais vulneráveis do ponto de vista ambiental e socioeconômico. Portanto, os programas e projetos de gestão ambiental e/ou territorial devem ser focados, primordialmente, na planície litorânea, nas planícies fluviais e lacustres, e na área de transição do tabuleiro pré-litorâneo com a depressão sertaneja.

Com relação à planície litorânea, averígua-se que toda a área deste compartimento geoambiental é classificada como de Média-Alta e Alta Vulnerabilidade Socioambiental. Dessa forma, merece dar ênfase ao setor de Alta Vulnerabilidade, que se estende da foz do Rio Caponga Funda até a foz do Rio Mal Cozinhado (Figura 8), que engloba as localidades de: Águas Belas, Caponga e Balbino, todas pertencentes ao município de Cascavel, estado do Ceará.

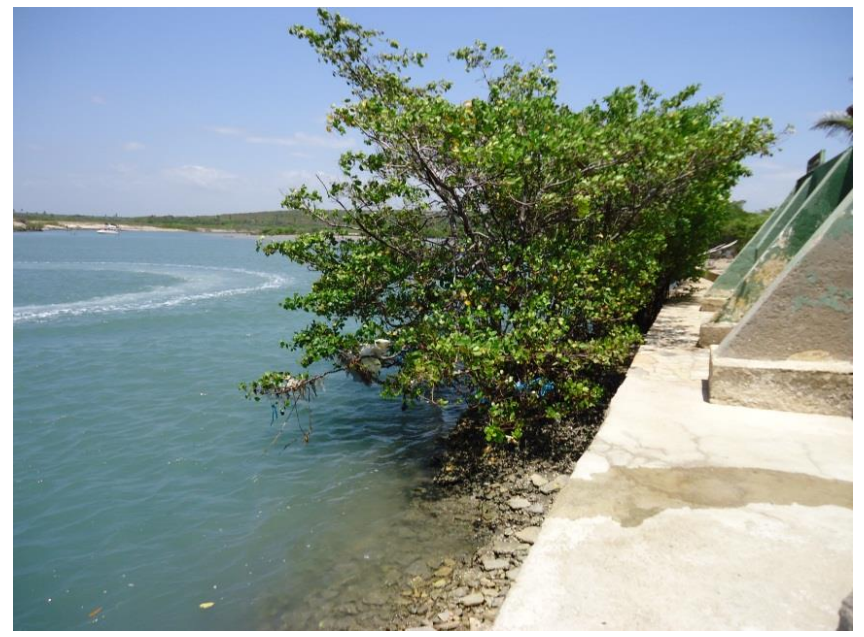

Figura 8: Estrangulamento antrópico da foz do Rio Mal Cozinhado, município de Cascavel (CE). Fonte: Autores (2014). Coordenada UTM / Datum SIRGAS 2000 / E=590.438; N=9.551.432.

Ao avaliar a vulnerabilidade socioambiental das planícies fluviais e lacustres, percebe-se que a mesma é classificada ora em Média-Alta Vulnerabilidade e ora em Alta Vulnerabilidade. Destaca-se que a alternância do IVSA é justificada pela caráter socioeconômico que se materializam de forma desigual no território, já que a 
ecodinâmica é uniforme para esse sistema ambiental. Com base no mapeamento realizado, constata-se que o IVSA é menor quanto mais próximo estar do perímetro urbano do município.

Em síntese, o Índice de Vulnerabilidade Socioambiental (IVSA) consiste em uma importante ferramenta orientativa de ordenamento territorial e de tomada de decisões por parte do poder público e da sociedade, ao indicar, de forma espacial, as condições socioeconômicas da população (sociedade) em consonância com a vulnerabilidade ambiental (natureza).

\section{Considerações Finais}

Ao considerar os municípios do setor leste da Região Metropolitana de Fortaleza (RMF) e, consequentemente, o recorte espacial das bacias hidrográficas costeiras dos rios Catú, Caburé, Caponda Funda, Caponga Roseira e Mal Cozinhado, constatam-se que essas áreas tiveram um acréscimo significativo em sua população nas últimas três décadas, influenciadas em grande parte, pela atração locacional proporcionada pela metrópole Fortaleza.

Diante deste cenário, é de fundamental importância agregar ao crescimento demográfico e ao avanço econômico a questão da melhoria da qualidade de vida das populações, incorporando na análise socioeconômica os princípios da preservação e conservação dos recursos naturais, por meio da concepção da sustentabilidade do desenvolvimento pelo viés socioambiental.

Ao combinar as informações de cunho ambiental e socioeconômica nas Unidades Hidrográficas de Planejamento (UHPs), constata-se uma intensa pressão antropogênica sobre os sistemas ambientais, com destaque especial para as planícies fluviais, lacustres e litorânea, tendo em vista que essas áreas foram apropriadas pelo modelo de desenvolvimento urbano/rural sem levar em consideração a ecodinâmica ambiental.

Como consequência da polarização das ações degradacionais, constata-se, dentre outros impactos ambientais negativos, a precariedade no sistema de saneamento básico, o gerenciamento inadequado dos resíduos sólidos, os desmatamentos indiscriminados, o assoreamento dos canais fluviais, entre outras variáveis inerentes às questões socioambientais verificadas in loco, o que vem ocasionando a agudização dos problemas socioambientais.

Com esse estudo, pretende-se contribuir para a construção de uma base teórico-metodológica e prática, proporcionando uma reflexão mais aprofundada a respeito da análise socioambiental como subsídio para o ordenamento territorial ao nível das UHPs. Com isso, almeja-se trabalhar e compatibilizar o desenvolvimento socioeconômico com a preservação da qualidade do meio ambiente e do equilíbrio ecológico, contemplando as diversas variáveis ambientais e socioeconômicos a partir da inter-relação entre sociedade e natureza. 


\section{Referências}

ALBUQUERQUE, E. L. S. Avaliação das condições socioambientais em bacias hidrográficas costeiras: contribuição ao ordenamento territorial do setor leste da região metropolitana de Fortaleza, Ceará. 2015. 258p. Tese (Doutorado em Geografia). Programa de Pós-Graduação em Geografia. Universidade Estadual do Ceará. Fortaleza: UECE, 2015.

ALBUQUERQUE, E. L. S.; MEDEIROS, C. N.; SOUZA, M. J. N. Geoinformação na abordagem de problemas socioambientais: aplicação do IDG no município de Horizonte, estado do Ceará. Revista Equador (UFPI), Vol. 4, N² 2, p. 128-144, 2015.

ALVES, H. P. F. Vulnerabilidade Socioambiental na Metrópole Paulistana: uma análise sociodemográfica das situações de sobreposição espacial de problemas e riscos sociais e ambientais. Revista Brasileira de Estudos da População, V.3, N 1, p. 43-59, 2005.

ANJOS, R. S. A. Dinâmica Territorial: Cartografia - Monitoramento - Modelagem. Brasília: Mapas Editora e Consultoria, 2008.

CUNHA, J. A. A gestão municipal através de tecnologia de geoprocessamento e cadastro urbano: Gerenciamento de dados físicos e sócio-econômicos do município de Serra Negra do Norte-RN. 2001. 101p. (Dissertação de Mestrado) - PPGeO, Universidade Federal do Rio Grande do Norte. Natal: UFRN, 2001.

DESCHAMPS, M. V. Vulnerabilidade Socioambiental na Região Metropolitana de Curitiba. Tese (Doutorado em Meio Ambiente e Desenvolvimento). 2004. Universidade Federal do Paraná. Curitiba: UFPR, 2004.

GRIGIO, A. M. Aplicação de sensoriamento remoto e sistema de informação geográfica na determinação da vulnerabilidade natural e ambiental do município de Guamaré (RN): simulação de risco às atividades da indústria petrolífera. 2003. 222p. (Dissertação de Mestrado). Programa de Pós-Graduação em Geodinâmica e Geofísica. Universidade Federal do Rio Grande do Norte. Natal: UFRN, 2003.

GUIMARÃES, L. T. Proposta de um sistema de Indicadores de Desenvolvimento Sustentável para as bacias hidrográficas. 2008. Tese (Doutorado em Engenharia). Programa de Pós-Graduação em Engenharia. Universidade Federal do Rio de Janeiro. Rio de Janeiro: UFRJ, 2008.

IBGE. Instituto Brasileiro de Geografia e Estatística. Censo Demográfico de 2010. 2010. Disponível em: www.sidra.ibge.gov.br. Acesso em: 25 jun. 2015.

LAURA, A. A. Um método de modelagem de um sistema de indicadores de sustentabilidade para gestão dos recursos hídricos - MISGERH: o caso da bacia dos Sinos. Tese (Doutorado em Engenharia de Recursos Hídricos e Saneamento Ambiental). Universidade Federal do Rio Grande do Sul. Porto Alegre: UFRGS, 2004.

MEDEIROS, C. N. Vulnerabilidade socioambiental do município de Caucaia (CE): Subsídios ao ordenamento territorial. 2014. 267p. Tese (Doutorado em Geografia). Programa de Pós-Graduação em Geografia. Universidade Estadual do Ceará. Fortaleza: UECE, 2014.

PEREIRA JÚNIOR, E. A. Industrialização e Reestruturação do Espaço Metropolitano. Fortaleza: Eduece, 2005. 
PNUD. Programa das Nações Unidas para o Desenvolvimento. Atlas do desenvolvimento humano no Brasil. 2013. Disponível em: http://www.atlasbrasil.org.br/2013. Acesso em: 24 Set. 2014.

\section{. Atlas do Desenvolvimento Humano nas Regiões Metropolitanas Brasileiras.}

Brasília: PNUD / Ipea / FJP, 2014.

SILVA, J. B. Características Gerais da Região Metropolitana de Fortaleza (RMF). In:

DANTAS, E; COSTA, M. C. L. Vulnerabilidade socioambiental na região

metropolitana de Fortaleza. Fortaleza: Edições UFC, 2009.

SOUZA, M. J. N. Bases naturais e esboço do zoneamento geoambiental do estado do Ceará. In: CRUZ, L. C.; SOUZA, M.J.N.; MORAES, J. O. (Orgs.) Compartimentação territorial e gestão regional do Ceará. Fortaleza: Ed. FUNECE, 2000. p.13-98.

TOMINAGA. L. K. Análise e Mapeamento de Risco In: TOMINAGA. L. K.; SANTORO, J; AMARAL, R. (orgs.) Desastres Naturais: conhecer para prevenir. São Paulo:

Instituto Geológico, p. 147-160, 2009.

TRICART, J. Ecodinâmica. Rio de Janeiro: IBGE/SUPREN, 1977. 91p.

ZANELLA, M.E; OLIMPIO, J. L. S; COSTA, M. C. L; DANTAS, E.W.C. Vulnerabilidade socioambiental do baixo curso da Bacia Hidrográfica do Rio Cocó, Fortaleza-CE. Revista Sociedade e Natureza, $\mathrm{n}^{\circ}$ 25, v. 2, p. 317-332. 2013.

\footnotetext{
Emanuel Lindemberg Silva Albuquerque

Doutor, Mestre em Geografia, Especialista em Geoprocessamento e Geógrafo pela Universidade Estadual do Ceará - UECE. Atualmente é Professor Adjunto I do Curso de Geografia da Universidade Federal do Piauí - UFPI

Universidade Federal do Piauí - UFPI Campus Universitário Ministro Petrônio Portella - Bairro Ininga - Teresina - PI. CEP: 64049-550.

E-mail: lindemberg@ufpi.edu.br

Cleyber Nascimento de Medeiros

Doutor em Geografia pelo Programa de Pós-Graduação em Geografia da Universidade Estadual do Ceará - UECE. Mestre em Geociências e Estatístico pela Universidade Federal do Rio Grande do Norte - UFRN. Atualmente é Analista de Políticas Públicas do Instituto de Pesquisa e Estratégia Econômica do Ceará - IPECE.

Av. Gen. Afonso Albuquerque Lima, s/n - Cambeba, Fortaleza - CE, 60822-325. E-mail: cleyber.medeiros@ipece.ce.gov.br
} 